\title{
Hyperinsulinaemia during exercise does not suppress hepatic glycogen concentrations in patients with type 1 diabetes: a magnetic resonance spectroscopy study
}

\author{
K. Chokkalingam $\cdot$ K. Tsintzas • J. E. M. Snaar • \\ L. Norton • B. Solanky • E. Leverton - P. Morris • \\ P. Mansell • I. A. Macdonald
}

Received: 20 February 2007 / Accepted: 28 May 2007 / Published online: 18 July 2007

(C) Springer-Verlag 2007

\begin{abstract}
Aims/hypothesis We compared in vivo changes in liver glycogen concentration during exercise between patients with type 1 diabetes and healthy volunteers.

MethodsWe studied seven men with type 1 diabetes (mean \pm SEM diabetes duration $10 \pm 2$ years, age $33 \pm 3$ years, BMI $24 \pm 1 \mathrm{~kg} / \mathrm{m}^{2}, \mathrm{HbA}_{1 \mathrm{c}} 8.1 \pm 0.2 \%$ and $V \mathrm{O}_{2}$ peak $43 \pm 2 \mathrm{ml}[\mathrm{kg}$ lean body mass $]^{-1} \mathrm{~min}^{-1}$ ) and five non-diabetic controls (mean \pm SEM age $30 \pm 3$ years, BMI $22 \pm 1 \mathrm{~kg} / \mathrm{m}^{2}, \mathrm{HbA}_{1 \mathrm{c}}$ $5.4 \pm 0.1 \%$ and $\mathrm{VO}_{2}$ peak $52 \pm 4 \mathrm{ml}[\mathrm{kg} \text { lean body mass }]^{-1}$ $\min ^{-1}$, before and after a standardised breakfast and after three bouts (EX1, EX2, EX3) of $40 \mathrm{~min}$ of cycling at $60 \%$ $\mathrm{VO}_{2}$ peak. ${ }^{13} \mathrm{C}$ Magnetic resonance spectroscopy of liver glycogen was acquired in a $3.0 \mathrm{~T}$ magnet using a surface coil. Whole-body substrate oxidation was determined using indirect calorimetry.
\end{abstract}

K. Chokkalingam and K. Tsintzas contributed equally to this study.

K. Chokkalingam $\cdot$ K. Tsintzas $\cdot$ L. Norton $\cdot$ I. A. Macdonald

Centre for Integrated Systems Biology and Medicine,

School of Biomedical Sciences, University of Nottingham,

Nottingham, UK

K. Chokkalingam $\cdot$ P. Mansell

Queen's Medical Centre,

Nottingham, UK

J. E. M. Snaar · B. Solanky · E. Leverton · P. Morris

Sir Peter Mansfield Magnetic Resonance Centre,

University of Nottingham,

Nottingham, UK

I. A. Macdonald ( $\square)$

School of Biomedical Sciences, Queen's Medical Centre,

University of Nottingham,

Derby Road,

Nottingham NG7 2UH, UK

e-mail: Ian.macdonald@nottingham.ac.uk
Results Blood glucose and serum insulin concentrations were significantly higher $(p<0.05)$ in the fasting state, during the postprandial period and during EX1 and EX2 in subjects with type 1 diabetes compared with controls. Serum insulin concentration was still different between groups during EX3 $(p<0.05)$, but blood glucose concentration was similar. There was no difference between groups in liver glycogen concentration before or after the three bouts of exercise, despite the relative hyperinsulinaemia in type 1 diabetes. There were also no differences in substrate oxidation rates between groups.

Conclusions/interpretation In patients with type 1 diabetes, hyperinsulinaemic and hyperglycaemic conditions during moderate exercise did not suppress hepatic glycogen concentrations. These findings do not support the hypothesis that exercise-induced hypoglycaemia in patients with type 1 diabetes is due to suppression of hepatic glycogen mobilisation.

Keywords Exercise $\cdot$ Hyperinsulinaemia $\cdot$ Liver glycogen metabolism · Magnetic resonance spectroscopy ·

Type 1 diabetes

Abbreviations
MRS magnetic resonance spectroscopy
RER
$V \mathrm{O}_{2} \quad$ oxpiratory exchange ratio

Introduction

Fear of exercise-induced hypoglycaemia poses a significant barrier to active participation in recreational sports and games in patients with type 1 diabetes [1-3]. The incidence 
of hypoglycaemia during an acute bout of moderateintensity exercise in children and adolescents with type 1 diabetes has been recently reported to be up to $30 \%$ [4]. Although there is no direct evidence, the incidence of exercise-induced hypoglycaemia can be expected to be similar in adults with type 1 diabetes. However, the physiological responses to exercise and hence the factors contributing to exercise-induced hypoglycaemia in patients with type 1 diabetes are not fully understood.

During exercise in non-diabetic individuals, either increased carbohydrate intake and/or increased hepatic glucose production is required to match the increased rate of muscle glucose uptake during exercise $[5,6]$. To facilitate increased hepatic glucose output, the hormonal response to exercise performed in the postabsorptive state is characterised by reciprocal changes in plasma insulin and glucagon concentrations [5]. Increases in both hepatic glycogenolysis and gluconeogenesis contribute to augmented hepatic glucose output during exercise, usually with a greater contribution from the former [5, 7]. However, during prolonged exercise under fasting conditions a greater contribution of hepatic glucose output is derived from gluconeogenesis $[8,9]$.

In patients with type 1 diabetes the hormonal and metabolic milieu is disturbed, and this may affect hepatic glycogen metabolism. First, it is clearly impossible to replicate the fall in insulin concentration normally seen during exercise using exogenous insulin injections in patients with type 1 diabetes. During exercise, systemic and to a lesser extent portal insulin concentrations will be higher in patients with type 1 diabetes treated with exogenous insulin than in normal subjects. Therefore, when patients exercise typically with high insulin concentrations $[3,10]$, this may suppress hepatic glucose output [11] with a consequent risk of hypoglycaemia both during and after exercise [11]. Indeed, infusing insulin during exercise in normal volunteers is associated with suppressed splanchnic glucose output [12]. Second, in addition to the fall in insulin, a rise in glucagon is required for the full increment in hepatic glucose output [13]. Prevention of this physiological response results in attenuation of hepatic glucose output [14]. It has also been suggested that the increased risk of hypoglycaemia in patients with type 1 diabetes could be due to impaired stimulation of hepatic glucose output in response to glucagon [15]. Third, patients with poorly controlled diabetes have low fasting hepatic glycogen concentrations and impaired net hepatic glycogen synthesis following ingestion of a mixed meal [16, 17]. This in turn may predispose these patients to hypoglycaemia during subsequent exercise.

A large body of evidence concerning hepatic glucose metabolism during exercise in diabetes has been derived from animal experiments [18-22] but there is a lack of in vivo human data. Human studies using magnetic resonance spectroscopy (MRS) have reported defects in mobilisation of hepatic glycogen stores in patients with type 1 diabetes $[23,24]$. However, these studies were performed either under very low circulating insulin concentrations (30$60 \mathrm{pmol} / \mathrm{l})$ or after hypoglycaemia. The impact of real-life therapeutic insulin concentrations on hepatic glycogen metabolism during exercise in patients with type 1 diabetes is not known. We have recently shown that, in patients with type 1 diabetes, exercise under peak therapeutic insulin concentrations increases exogenous glucose utilisation and that this increase is disproportionate to the relative increase in carbohydrate oxidation, suggesting an increase in glucose flux through non-oxidative pathways, perhaps towards hepatic glycogenesis [25].

The aim of this study was to compare in vivo changes in liver glycogen both before and after a meal, and during subsequent moderate exercise in patients with type 1 diabetes under therapeutic insulin concentrations, and in non-diabetic volunteers. In line with current recommendations $[2,10]$, we used a $50 \%$ reduction of the usual preprandial rapid-acting insulin dose. In addition, rapidacting insulin was infused intravenously to achieve a predictable basal replacement $(\sim 0.5 \mathrm{U} / \mathrm{h})$. Although clearly not truly real-life, we adopted this strategy to approximately replicate a real-life situation. We hypothesised that, when compared with non-diabetic controls, patients with type 1 diabetes when exercising under relative hyperinsulinaemic conditions may suppress hepatic glycogen mobilisation and thereby increase the risk of exercise-induced hypoglycaemia.

\section{Methods}

Patients and preliminary measurements We recruited seven physically active men with uncomplicated type 1 diabetes from our hospital diabetes clinics, with mean \pm SEM diabetes duration $10 \pm 2$ years, and five non-diabetic, physically active controls. The baseline characteristics of the volunteers are outlined in Table 1. Fat-free mass was determined using a bioelectrical impedance technique (Quadscan 4000; Bodystat, Isle of Man, UK). Five patients were on a basal-bolus subcutaneous insulin regimen and two were on twice a day premixed insulin injections. All patients were screened to exclude micro- and macrovascular complications. The control subjects were medically screened and were in good health. Patients and controls were informed of all experimental procedures and the associated risks before we obtained informed consent. All procedures used in this study were performed according to the Declaration of Helsinki and approved by the Nottingham NHS Research Ethics Committee and the University of Nottingham Research Ethics Committee. 
Table 1 Baseline characteristics of study participants

\begin{tabular}{lccc}
\hline & Type 1 diabetes $(n=7)$ & Controls $(n=5)$ & $p$ value \\
\hline Age $($ years $)$ & $33 \pm 3$ & $30 \pm 3$ & 0.509 \\
$\mathrm{BMI}\left(\mathrm{kg} / \mathrm{m}^{2}\right)$ & $24 \pm 1$ & $22 \pm 1$ & 0.054 \\
Lean body mass $(\mathrm{kg})$ & $68 \pm 3$ & $65 \pm 5$ & 0.548 \\
$\mathrm{HbA}_{1 \mathrm{c}}(\%)$ & $8.1 \pm 0.2$ & $5.4 \pm 0.1$ & 0.000 \\
$V \mathrm{O}_{2}$ peak $\left(\mathrm{ml}\left[\mathrm{kg}\right.\right.$ lean body mass $\left.{ }^{-1} \mathrm{~min}^{-1}\right)$ & $43 \pm 2$ & $52 \pm 4$ & 0.09
\end{tabular}

Data are presented as means \pm SEM

The peak oxygen uptake $\left(\mathrm{VO}_{2}\right.$ peak) was determined during a preliminary visit using an incremental cycling test (Excalibur cycle ergometer; Lode, Groningen, the Netherlands). The criteria used for attainment of the $V \mathrm{O}_{2}$ peak were: heart rate response greater than $90 \%$ of predicted maximum heart rate, respiratory exchange ratio (RER) $>$ 1.10 , near maximal rating of perceived exertion (Borg scale $\geq 19)$ and a drop in pedalling rate greater than $20 \%$.

Experimental design and protocol An outline of the study design is depicted in Fig. 1. For 3 days prior to the trials, controls and patients maintained an isoenergetic diet and patients monitored their capillary blood glucose closely to prevent episodes of hypoglycaemia. All participants were requested to avoid smoking, alcohol and exercise for 3 days prior to each study visit.

Patients (type 1 diabetes) omitted their medium-/longacting insulin for $24-36 \mathrm{~h}$ prior to the study. On the evening prior to the study, all subjects consumed a standardised high-carbohydrate $(2.5 \mathrm{~g} / \mathrm{kg}$ fat-free mass $)$ meal, and the patients injected their usual short-acting soluble insulin with this meal and were admitted to the hospital. An antegrade cannula was inserted into a cubital fossa vein for a low-dose intravenous insulin infusion to maintain euglycaemia overnight. A retrograde cannula was inserted into a dorsal hand vein for blood sampling to measure glucose concentrations during the overnight stay. On the day of the study this cannula was also used for drawing arterialised blood samples for metabolite and hormone measurements, while the hand was placed in a hot air box $\left(55^{\circ} \mathrm{C}\right)$. At 08:00 hours the following morning, subjects were transferred to the magnetic resonance (MR) centre for the study protocol. Intravenous insulin infusion was continued at a constant rate of $5 \mathrm{mU} \mathrm{m} \mathrm{min}^{-1}$ to cover basal insulin requirements until the end of the study.

The non-diabetic controls reported directly to the MR centre after an overnight fast from 22:00 hours and a retrograde cannula was inserted into a dorsal hand vein for drawing arterialised blood samples as above. A baseline ${ }^{13} \mathrm{C}$ MRS measurement of liver glycogen was carried out for both patients and controls. All subjects were then provided with a high-glycaemic index (82.9) carbohydrate meal $(2.5 \mathrm{~g}$ carbohydrate $/ \mathrm{kg}$ fat-free mass $)$. The meal consisted of Kellogg's corn flakes, skimmed milk, white bread, strawberry jam and Lucozade (Glaxo SmithKline, Brentford, UK). The diabetic subjects received a subcuta-

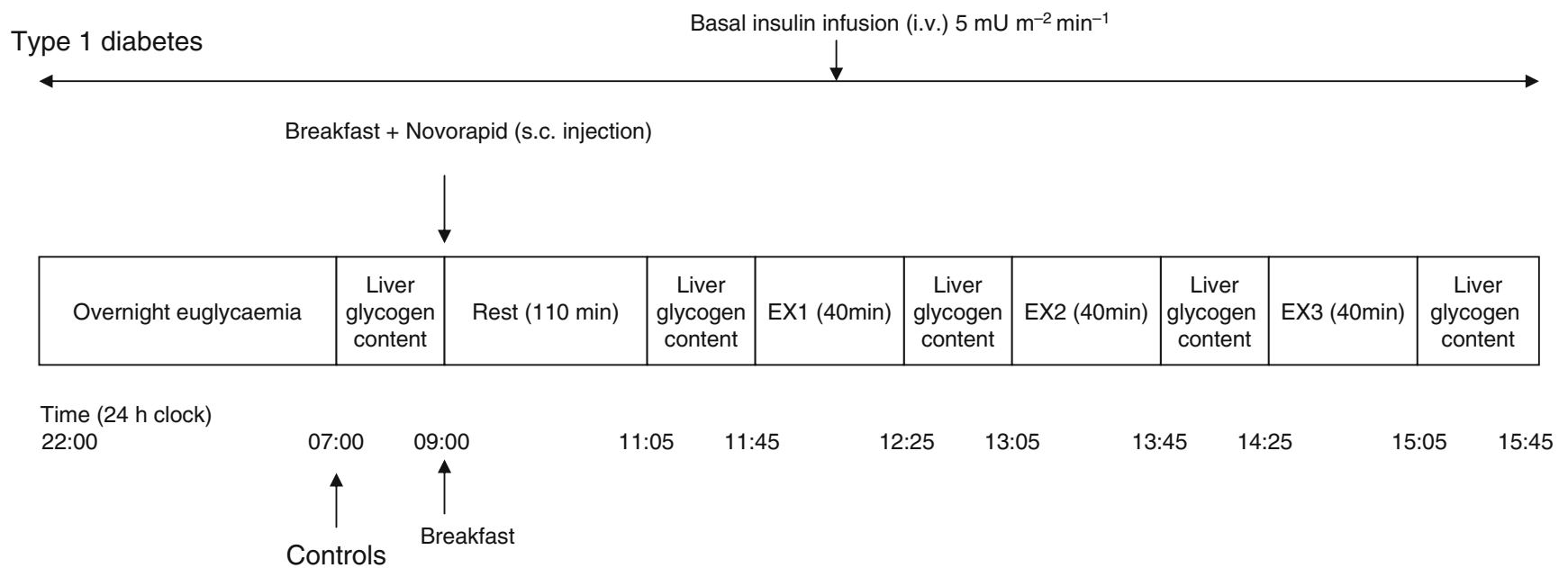

Fig. 1 Schematic diagram of study design. EX1, 2 and 3 represent the three bouts of exercise. Blood samples were drawn at regular intervals during the study for metabolite and hormone measurements. Indirect calorimetry was carried out during a separate visit under identical feeding and exercise conditions, before and after breakfast and during exercise, to measure substrate oxidation rates. There was a slight variation in the insulin treatment protocol when data were acquired (see Methods) 
neous abdominal injection of $0.5 \mathrm{U}$ of quick-acting analogue insulin (Novorapid; Novo Nordisk, Chartres, France) per $10 \mathrm{~g}$ of carbohydrate with breakfast (a $50 \%$ dose reduction from what these patients would have normally administered for this meal). All subjects were requested to consume the meal within $15 \mathrm{~min}$ and subsequently rested on a bed for $110 \mathrm{~min}$ to replicate a typical postprandial pre-exercise state. Further spectroscopy measurements of liver glycogen were acquired $110 \mathrm{~min}$ after consumption of the meal. All subjects (subjects with type 1 diabetes and controls) then carried out three bouts of cycling at $60 \% V \mathrm{O}_{2}$ peak for $40 \mathrm{~min}$ each. The cycling sessions were interrupted for $40 \mathrm{~min}$ between bouts to obtain further spectroscopy measurements of liver glycogen. Hydration was maintained during cycling by ingesting water at a rate of $10 \mathrm{ml} / \mathrm{kg}$ body weight every $20 \mathrm{~min}$. Heart rate and rating of perceived exertion using a Borg scale [26] were measured at $20 \mathrm{~min}$ intervals during exercise. Blood samples were drawn at the same intervals for metabolite and hormone measurements. Urine samples were collected throughout the experiment for glucose measurements.

On a separate day to the liver glycogen data acquisition visit, the above exercise and feeding protocol was replicated in each non-diabetic volunteer and patient with diabetes to acquire indirect calorimetry data. The presence of a strong magnetic field and working space limitations precluded us from making these measurements on the same visit as when MR scanning was taking place. During this visit there was a slight variation in the insulin treatment protocol. On the evening prior to this visit, patients had the same high-carbohydrate meal and their usual insulin treatment regime (quick-acting insulin followed by their long-acting insulin). Patients were advised to monitor their capillary glucose regularly to avoid hypoglycaemia and were not admitted overnight. Moreover, they did not receive the intravenous basal insulin infusion during the study since patients administered their usual long-acting insulin the previous night. Resting $V \mathrm{CO}_{2}$ and $V \mathrm{O}_{2}$ were measured for 20 min using a ventilated hood system (GEM; Nutren Technologies, Manchester, UK) before and $110 \mathrm{~min}$ after consumption of the same meal to allow calculation of energy expenditure and substrate oxidation rates [27]. Subsequently, $5 \mathrm{~min}$ expired gas samples (Vmax 29; Sensormedics, Yorba Linda, CA, USA) were collected every $20 \mathrm{~min}$ whilst the subject was cycling and the measured $V \mathrm{CO}_{2}$ and $V \mathrm{O}_{2}$ values were used to determine substrate oxidation rates during exercise.

Blood and urine metabolites Whole-blood and urine glucose and blood lactate were measured using a Yellow Springs analyser (YSI 2300 Stat Plus-D; Yellow Springs Instruments, Yellow Springs, OH, USA). All serum and plasma samples were stored at $-80^{\circ} \mathrm{C}$ until analysis. Serum insulin and glucagon were measured using commercially available radioimmunoassay kits from Diagnostic Products Corporation (Llanberis, UK). NEFA concentrations were measured using a kit from Wako Chemicals (Neuss, Germany). Whole-blood $\beta$-hydroxybutyrate was measured in blood samples treated with perchloric acid (10\%) [28]. Plasma catecholamines were measured using HPLC with electrochemical detection [29].

${ }^{13} \mathrm{C}$ Magnetic resonance spectroscopy $\mathrm{All}{ }^{13} \mathrm{C}$ MR spectra were acquired on a $3.0 \mathrm{~T}$ whole-body magnetic resonance scanner with a $1 \mathrm{~m}$ diameter bore. A surface coil was used with a carbon coil for transmission and reception, and quadrature proton coils for ${ }^{1} \mathrm{H}$ decoupling. This consisted of a $7 \mathrm{~cm}$ diameter circular ${ }^{13} \mathrm{C}$ coil and two $13 \mathrm{~cm}{ }^{1} \mathrm{H}$ coils. Subjects were positioned in the scanner in the supine position with the surface coil placed over the liver region of the torso. Care was taken to reposition subjects and the coil as accurately as possible for subsequent measurements. Manual shimming was performed on the water resonance and the broadband decoupling frequency was centred on the glycogen resonance. The coils were all tuned and matched using a Network Analyser (Model 8751A; Hewlett-Packard; 5-500 kHz). ${ }^{13} \mathrm{C}$ spectra were acquired with a simple pulse-acquire (decouple) sequence. The excitation pulse was a $100 \mu$ s pulse at a peak power of 390 (10) W with Cyclops phase cycling. Broadband decoupling was achieved using three WALTZ- 8 cycles during acquisition with a peak power of $50 \pm 2$ W. A repetition time of $360 \mathrm{~ms}$ was used and spectra were collected in blocks of 1000 acquisitions, giving a temporal resolution of $6 \mathrm{~min}$. Two blocks were collected and summed for each time point. The sampling time was $142 \mu$ s, and 512 data points were collected during the acquisition time. RF powers were monitored throughout the acquisition period to ensure that we did not exceed the maximum values allowed according to specific absorption rate limits recommended by the National Radiological Protection Board. No adjustments to the spectrometer were made between measurements, except for tuning and matching the ${ }^{13} \mathrm{C}$ surface coil before each spectroscopic measurement.

All spectra were analysed using the Matlab version of the magnetic resonance user interface (MRUI). Spectral peaks were selected using the AMARES algorithm and were fitted to Lorentzian lineshapes. The signal of interest arises from the $\mathrm{C} 1$ position of glycogen at $100.5 \mathrm{ppm}$ (relative to tetramethylsilane $=0$ ). The lipid peaks obscured resonances from other glycogen carbons (C2 to C6). The coefficient of variation, calculated for repeated analysis of a single spectrum, was $3.8 \%(n=15)$. The integral of the glycogen peak was expressed as a fraction of the formate peak derived from a phantom containing formate at the centre of the coil. Quantification was done using a liver-shaped phantom 
containing a solution of $184 \mathrm{mmol} / \mathrm{l}$ oyster glycogen and $150 \mathrm{mmol} / \mathrm{l} \mathrm{KCl}$. Glycogen concentrations were calculated using the formula: $\left(R_{\mathrm{gly}(\mathrm{s})} \times[\mathrm{Glyc}]\right) / R_{\mathrm{gly}(\mathrm{ph})}$, where $R_{\mathrm{gly}(\mathrm{ph})}$ and $R_{\mathrm{gly}(\mathrm{s})}$ are the ratios of glycogen to formate in the phantom and subject, respectively, and [Glyc] is the concentration of glycogen in the phantom in $\mathrm{mmol} / \mathrm{l}$.

Statistics Statistical analysis was carried out using SPSS version 11.5. A mixed-factor ANOVA was used to compare data from repeated measures. A $p$ value of $<0.05$ was considered significant. All data are expressed as mean \pm SEM.

\section{Results}

Patients and controls cycled at a mean workload of $153 \pm 12$ and $169 \pm 17 \mathrm{~W}$. The respective mean oxygen consumptions were $26.6 \pm 1.11$ and $29.8 \pm 2.5 \mathrm{ml}$ [kg lean body mass] ${ }^{-1}$ $\min ^{-1}$, being almost $60 \%$ of the predetermined $V_{2}$ peak. Two patients developed hypoglycaemic symptoms (blood glucose $<2.5 \mathrm{mmol} / \mathrm{l}$ ) at the end of the second bout of exercise and did not proceed to the third bout. A further two patients developed asymptomatic hypoglycaemia (defined as blood glucose $<3.5 \mathrm{mmol} / \mathrm{l}$ ) at the end of the third bout of exercise.

Blood metabolites and hormones Blood glucose concentrations were higher in patients than controls at rest and during EX1 and EX2 (Fig. 2a, $p<0.05$ ) but similar at the end of EX2 and during EX3. Fasting serum insulin concentration was greater in patients than controls (Fig. 2b, $p<0.05$ ). During all three bouts of exercise, serum insulin concentrations were greater in patients than controls (Fig. 2b, $p<0.05$ ). Fasting plasma NEFA concentrations were comparable between groups. Plasma NEFA concentrations were suppressed after breakfast and during EX1 in both groups (Table 2). NEFA concentrations started rising during EX2 and peaked during EX3 $(p<0.05)$, with no differences between groups. There was no difference in blood $\beta$-hydroxybutyrate concentrations between groups during the entire experiment (Table 2). Fasting blood lactate concentrations were also comparable between groups (Table 2). Exercise induced a significant $(p<0.05)$ increase in blood lactate concentrations in both groups, with no significant differences between groups. Baseline plasma noradrenaline and adrenaline concentrations were comparable in the two groups (Table 2). Exercise induced a significant $(p<0.05)$ increase in both plasma adrenaline and noradrenaline concentrations in both groups during EX1, EX2 and EX3, with no difference between groups. Fasting serum glucagon concentrations were significantly $(p<0.05)$ lower in patients with type 1 diabetes compared with
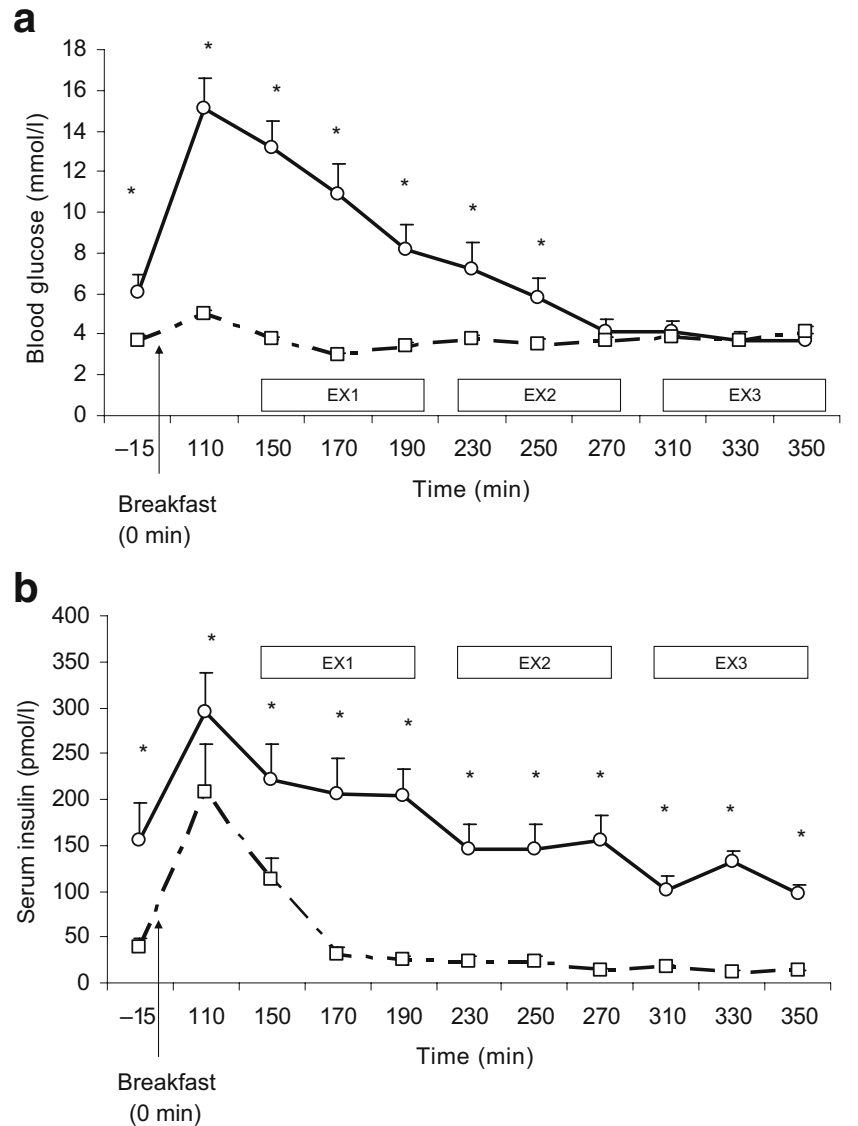

Fig. 2 Blood glucose (a) and serum insulin (b) concentrations in patients with type I diabetes (circles) and controls (squares). EX1, 2 and 3 represent the first, second and third bouts of exercise, respectively. ${ }^{*} p<0.05$ for differences in blood glucose and serum insulin concentrations vs controls. $n=7$ and $n=5$ in the patient and control groups, respectively, except $n=5$ at time points 330 and $350 \mathrm{~min}$ in the patient group

controls (Table 2). Following the meal there was a significant $(p<0.05)$ increase in serum glucagon concentrations in patients with diabetes but not in controls, resulting in similar pre-exercise values in the two groups. Furthermore, there was no difference in glucagon concentrations during exercise between groups.

Whole-body substrate metabolism There were no differences in either fasting or postprandial RER values between patients and controls (Table 3). A substantial increase in whole body carbohydrate oxidation was observed with the onset of exercise but there was no difference between groups (Table 3). Similarly, a substantial increase in wholebody fat oxidation was observed during exercise, again with no differences between groups (Table 3).

Liver glycogen There were no significant differences in fasting liver glycogen between groups (type 1 diabetes, 
Table 2 Blood metabolite and hormone concentrations before and after breakfast and at the end of each exercise session

\begin{tabular}{|c|c|c|c|c|c|c|c|c|c|c|}
\hline & \multicolumn{2}{|l|}{ Fasting } & \multicolumn{2}{|l|}{ After meal } & \multicolumn{2}{|l|}{ End of EX1 } & \multicolumn{2}{|l|}{ End of EX2 } & \multicolumn{2}{|l|}{ End of EX3 } \\
\hline & $\begin{array}{l}\text { Type } 1 \\
\text { diabetes }\end{array}$ & Controls & $\begin{array}{l}\text { Type } 1 \\
\text { diabetes }\end{array}$ & Controls & $\begin{array}{l}\text { Type } 1 \\
\text { diabetes }\end{array}$ & Controls & $\begin{array}{l}\text { Type } 1 \\
\text { diabetes }\end{array}$ & Controls & $\begin{array}{l}\text { Type } 1 \\
\text { diabetes }\end{array}$ & Controls \\
\hline $\begin{array}{l}\text { Plasma NEFA } \\
(\mathrm{mmol} / \mathrm{l})\end{array}$ & $0.31 \pm 0.05$ & $0.32 \pm 0.07$ & $0.05 \pm 0.02 *$ & $0.06 \pm 0.01 *$ & $0.05 \pm 0.02 *$ & $0.06 \pm 0.02 *$ & $0.45 \pm 0.17$ & $0.48 \pm 0.10$ & $0.87 \pm 0.13^{*}$ & $1.29 \pm 0.18^{*}$ \\
\hline $\begin{array}{l}\text { Blood } \beta \text { - } \\
\text { hydroxybutyrate } \\
(\mathrm{mmol} / \mathrm{l})\end{array}$ & $0.32 \pm 0.19$ & $0.05 \pm 0.01$ & $0.06 \pm 0.01$ & $0.02 \pm 0.01$ & $0.10 \pm 0.01$ & $0.09 \pm 0.01$ & $0.06 \pm 0.01$ & $0.08 \pm 0.02$ & $0.46 \pm 0.12$ & $0.42 \pm 0.18$ \\
\hline $\begin{array}{l}\text { Blood lactate } \\
(\mathrm{mmol} / \mathrm{l})\end{array}$ & $1.0 \pm 0.2$ & $0.9 \pm 0.1$ & $1.2 \pm 0.1$ & $1.0 \pm 0.1$ & $2.5 \pm 0.6^{*}$ & $1.6 \pm 0.3^{*}$ & $1.9 \pm 0.3^{*}$ & $1.2 \pm 0.2^{*}$ & $1.6 \pm 0.3 *$ & $1.2 \pm 0.2^{*}$ \\
\hline $\begin{array}{l}\text { Plasma noradrenaline } \\
(\mathrm{nmol} / \mathrm{l})\end{array}$ & $3.4 \pm 0.2$ & $2.8 \pm 0.3$ & $2.0 \pm 0.1$ & $2.3 \pm 0.2$ & $9.4 \pm 0.6^{*}$ & $8.5 \pm 1.1^{*}$ & $7.8 \pm 1.6^{*}$ & $8.9 \pm 1.1^{*}$ & $10.5 \pm 1.1^{*}$ & $8.0 \pm 1.6^{*}$ \\
\hline $\begin{array}{l}\text { Plasma adrenaline } \\
(\mathrm{nmol} / \mathrm{l})\end{array}$ & $0.2 \pm 0.1$ & $0.1 \pm 0.03$ & $0.2 \pm 0.04$ & $0.2 \pm 0.03$ & $1.1 \pm 0.2 *$ & $1.4 \pm 0.5^{*}$ & $2.2 \pm 0.8^{*}$ & $2.2 \pm 1.0^{*}$ & $5.7 \pm 1.6^{*}$ & $2.1 \pm 0.5^{*}$ \\
\hline $\begin{array}{l}\text { Serum glucagon } \\
(\mathrm{pg} / \mathrm{ml})\end{array}$ & $50.3 \pm 3.1$ & $70.9 \pm 9.4^{* *}$ & $78.6 \pm 5.0$ & $77.4 \pm 17.5$ & $77.5 \pm 6.6^{*}$ & $90.2 \pm 14.1^{*}$ & $79.1 \pm 5.0^{*}$ & $87.4 \pm 12.9^{*}$ & $78.3 \pm 3.9^{*}$ & $92.0 \pm 11.5^{*}$ \\
\hline
\end{tabular}

Data are presented as means \pm SEM

Type 1 diabetes $n=7$ and controls $n=5$ for all metabolites and hormones except $\beta$-hydroxybutyrate ( $n=4$ both groups) and for all metabolites and hormones during $\mathrm{EX} 3$ ( $n=5$ in the patient group)

${ }^{*} p<0.05$ for difference from fasting value within the same trial

$* * p<0.05$ compared with type 1 diabetes

$178 \pm 54 \mathrm{mmol} / \mathrm{l}$; controls, $212 \pm 63 \mathrm{mmol} / \mathrm{l}$; Fig. 3). Following the high-carbohydrate breakfast, there was a two- to threefold increase in hepatic glycogen concentration in both patients and controls but there was no difference between groups (type 1 diabetes, $589 \pm 138 \mathrm{mmol} / \mathrm{l}$; controls, $463 \pm 77$ mmol/1; Fig. 3). Hepatic glycogen concentrations decreased after EX1 (type 1 diabetes, $298 \pm 67 \mathrm{mmol} / \mathrm{l}$; controls, $303 \pm 59 \mathrm{mmol} / \mathrm{l}$ ), EX2 (type 1 diabetes, $267 \pm 41 \mathrm{mmol} / \mathrm{l}$; controls, $210 \pm 51 \mathrm{mmol} / \mathrm{l}$ ) and EX3 (type 1 diabetes, $195 \pm 80 \mathrm{mmol} / \mathrm{l}$; controls, $145 \pm 63 \mathrm{mmol} / \mathrm{l}$ ) in both groups (Fig. 3). There was no difference in hepatic glycogen concentrations during exercise between patients and controls.

\section{Discussion}

There were three main findings of this study. First, following a standardised evening meal and overnight control of glycaemia, fasting hepatic glycogen concentrations in patients with type 1 diabetes were comparable with

Table 3 RER and substrate oxidation rates before and after breakfast and during exercise

\begin{tabular}{|c|c|c|c|c|c|c|}
\hline & \multicolumn{2}{|c|}{$\begin{array}{l}\text { Carbohydrate oxidation } \\
\left(\mathrm{mg} \min ^{-1}[\mathrm{~kg} \text { lean body mass }]^{-1}\right)\end{array}$} & \multicolumn{2}{|c|}{$\begin{array}{l}\text { Fat oxidation } \\
\left(\operatorname{mg} \min ^{-1}[\mathrm{~kg} \text { lean body mass }]^{-1}\right)\end{array}$} & \multicolumn{2}{|l|}{ RER } \\
\hline & Type 1 diabetes & Controls & Type 1 diabetes & Controls & Type 1 diabetes & Controls \\
\hline Fasting & $0.17 \pm 0.06$ & $0.28 \pm 0.04$ & $0.14 \pm 0.02$ & $0.09 \pm 0.02$ & $0.79 \pm 0.03$ & $0.87 \pm 0.03$ \\
\hline $1 \mathrm{~h}$ after breakfast & $0.44 \pm 0.07$ & $0.49 \pm 0.10$ & $0.06 \pm 0.02$ & $0.06 \pm 0.05$ & $0.91 \pm 0.03$ & $0.94 \pm 0.05$ \\
\hline $2 \mathrm{~h}$ after breakfast & $0.46 \pm 0.03$ & $0.55 \pm 0.06$ & $0.06 \pm 0.01$ & $0.02 \pm 0.03$ & $0.93 \pm 0.02$ & $0.98 \pm 0.03$ \\
\hline EX1 & $3.47 \pm 0.16$ & $3.59 \pm 0.23$ & $0.49 \pm 0.04$ & $0.50 \pm 0.06$ & $0.92 \pm 0.01$ & $0.92 \pm 0.01$ \\
\hline EX2 & $3.11 \pm 0.20$ & $3.78 \pm 0.28$ & $0.56 \pm 0.06$ & $0.43 \pm 0.12$ & $0.90 \pm 0.01$ & $0.93 \pm 0.02$ \\
\hline EX3 & $2.92 \pm 0.31$ & $3.21 \pm 0.17$ & $0.72 \pm 0.13$ & $0.73 \pm 0.10$ & $0.88 \pm 0.02$ & $0.89 \pm 0.01$ \\
\hline
\end{tabular}

Data are presented as means \pm SEM

EX1, 2 and 3 represent the first, second and third bouts of exercise, respectively

Values for EX1, EX2 and EX3 represent the average during each bout of exercise

$n=7$ and $n=5$ in the patient and control groups, respectively, except $n=6$ during EX 3 in the patient group 


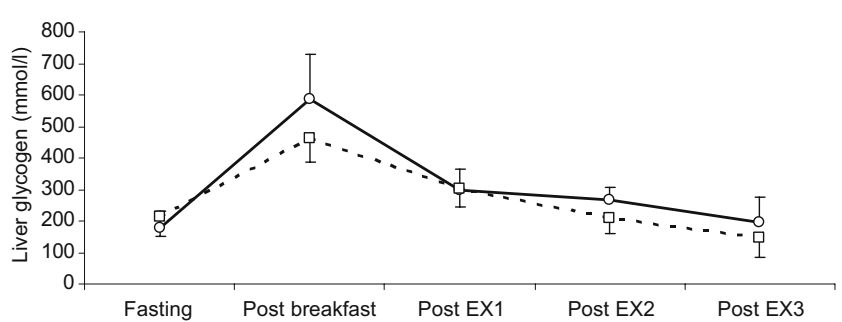

Fig. 3 Liver glycogen concentrations in patients with type 1 diabetes (circles) and controls (squares) before and after a standardised breakfast, and after three bouts of $40 \mathrm{~min}$ of submaximal exercise. EX1, 2 and 3 represent the first, second and third bouts of exercise, respectively. $n=7$ and $n=5$ in the patient and control groups, respectively, except $n=5$ after EX3 in the patient group

those in non-diabetic controls. Second, similar postprandial liver glycogen deposition occurred after consumption of a high-glycaemic index breakfast and injection of a lower than normal dose of subcutaneously administered rapidacting insulin analogue. Third, changes in liver glycogen content during exercise were similar in patients with type 1 diabetes and in controls despite the higher relative systemic hyperinsulinaemia (threefold) present in the former compared with the latter group.

Insulin, glucagon and catecholamines respond in a hierarchical fashion to regulate hepatic glucose output and prevent exercise-induced hypoglycaemia [14, 30-32]. In non-diabetic individuals a fall in serum insulin concentration and an increase in glucagon occur during exercise. This allows increased hepatic glucose output to match the increased muscle glucose uptake which occurs during exercise [33]. In patients with type 1 diabetes managed with subcutaneous insulin injections, a fall in serum insulin concentration cannot occur during exercise. Hence, if the physiological augmentation of hepatic glucose output is thereby inhibited, the blood glucose concentration may fall. However, the present study shows that the change in liver glycogen content during exercise in patients with type 1 diabetes was comparable to that of the non-diabetic controls despite the prevailing hyperinsulinaemic, hyperglycaemic conditions. This is in contrast to previous studies in healthy humans [24] and some [34] but not all animal studies [22, 35], which demonstrated insulin-induced suppression of hepatic glucose output. It could be argued that, despite the prevailing hyperinsulinaemic conditions, a preserved glucagon response to exercise could have accounted for the lack of differences in exercise-induced changes in liver glycogen content between groups. Indeed, a rise in glucagon is required during postabsorptive exercise for the full increment in hepatic glucose output $[8,13]$. On the contrary, it has been shown in patients with type 1 diabetes that, during exercise under fasting conditions, hepatic glucose output is comparable to that of controls, and this is despite higher circulating insulin concentrations and lower glucagon responses in the patient group [36]. The role of glucagon in the regulation of hepatic glucose output during exercise performed in the postprandial state is not clear. We have recently shown that moderate intensity exercise performed during a hyperinsulinaemic clamp does not induce a significant increase in glucagon concentrations in patients with type 1 diabetes [25]. Furthermore, in the present study there was no difference in serum glucagon concentrations during exercise performed in the postprandial state between patients with type 1 diabetes and controls. This resulted in a lower ratio of glucagon to insulin in the patient group when compared with the control group. Therefore, the parallel reduction in hepatic glycogen content between groups despite the obvious differences in the glucagon/insulin ratio suggests that other factors are important for the regulation of hepatic glucose output during exercise performed in the postprandial state in patients with type 1 diabetes. It is plausible that a preserved exercise-induced increase in circulating catecholamines rather than the glucagon response enabled continuous substrate mobilisation from the liver in this patient cohort $[14,30,36-38]$. It is known that pre-exercise hypoglycaemia is associated with blunted counter-regulation and impaired hepatic glycogenolysis [23, 39]. Since in the present study, patients were admitted overnight and hypoglycaemia was avoided prior to the experiment, counter-regulatory mechanisms were likely to have been preserved. Hence, counterregulatory mechanisms may play a more prominent role in hepatic glycogen metabolism during exercise than the suppressive effect of relative hyperinsulinaemia in patients with type 1 diabetes.

Hepatic carbohydrate metabolism has been reported to be defective in patients with poorly controlled type 1 diabetes $[16,17]$. It is likely that portal hypoinsulinaemia due to poorly controlled type 1 diabetes contributed to the lower fasting hepatic glycogen concentrations observed in these studies [21]. Since in the present study the patients were infused with intravenous insulin overnight, portal hypoinsulinaemia would have been alleviated, which may explain why we did not observe any difference in fasting glycogen concentrations. Patients with poorly controlled type 1 diabetes have impaired net hepatic glycogen synthesis following ingestion of a mixed meal and show an increase in hepatic gluconeogenesis [17]. However, these defects can be corrected after combined long-term and overnight control of glycaemia with intravenous insulin [40], and our results support this observation. Postprandial, pre-exercise liver glycogen concentration in type 1 diabetes was comparable to that in control subjects despite the relative lower estimated portal insulin concentration $(\sim 150$ $200 \mathrm{pmol} / \mathrm{l})$ when compared with controls ( 600 pmol/l). This is most likely to be the result of hyperglycaemic conditions in the postprandial period. Combined hyperglycaemic and hyperinsulinaemic conditions result in 
increased hepatic glycogen synthesis as a result of the stimulatory effect on glycogen synthase flux and inhibition of glycogen phosphorylase [41].

Previous studies demonstrated a preferential utilisation of NEFAs over carbohydrate in patients with type 1 diabetes during moderate exercise in the fasting state [42, 43]. However, these experiments were performed after insulin withdrawal for $24 \mathrm{~h}$. When moderate exercise was performed with insulin replacement ( $65 \mathrm{pmol} / \mathrm{l})$, patients with type 1 diabetes had comparable carbohydrate but higher fat oxidation rates when compared with controls $[11,36]$. It is likely that, in the present study, the higher circulating systemic insulin concentrations $(\sim 150 \mathrm{pmol} / \mathrm{l})$ corrected the previously reported differences in substrate oxidation between patients with type 1 diabetes and controls.

A typical carbohydrate-rich pre-exercise meal and a reduced subcutaneous insulin dose were used in this study. The patients were hyperglycaemic at the start of exercise and, despite the $50 \%$ insulin dose reduction, patients were still hyperinsulinaemic $(>200 \mathrm{pmol} / \mathrm{l})$. Routine clinical care would recommend that patients do not carry out exercise at these levels of hyperglycaemia for fear of inducing diabetic ketoacidosis. Our data demonstrate that as long as adequate insulinisation is maintained the risk of inducing significant ketosis is minimal. However, there is a dearth of clinical evidence as to the ideal glycaemic level at which patients should exercise to derive both health benefits and prevent exercise-induced hypoglycaemia. We observed a greater fall in blood glucose concentration (from 13 to $8 \mathrm{mmol} / \mathrm{l}$ ) during EX1, whereas only a modest fall (from 8 to $5 \mathrm{mmol} / \mathrm{l}$ ) was observed during EX2 and little or no change during EX3. It is likely that this was due to the combined effect of hyperglycaemia and a muscle contraction-induced increase in glucose disposal.

We have recently shown that exercise under differing insulin concentrations ( $\sim 150$ vs $\sim 500 \mathrm{pmol} / \mathrm{l})$ is associated with similar energy expenditure and muscle glycogen utilisation despite substantial increase in exogenous glucose utilisation [25]. Hence, we suggested that glucose was possibly being diverted during exercise to non-oxidative pathways in the liver and/or non-contracting muscle. However, in the present study, contrary to our hypothesis, we did not observe any sparing of hepatic glycogen utilisation in patients with type 1 diabetes under hyperinsulinaemic conditions when compared with controls. Interestingly, four patients developed hypoglycaemia at the end of the second and third bouts of exercise despite the lack of suppression of hepatic glycogen utilisation. This was possibly related to either the duration of exercise [44], the suppression of gluconeogenesis at these high insulin concentrations or differences in the relative contributions of blood glucose and muscle glycogen to muscle carbohydrate oxidation during the second and third bouts of exercise.
In conclusion, in patients with type 1 diabetes, hyperinsulinaemic and hyperglycaemic conditions during moderate exercise did not suppress changes in hepatic glycogen concentration when compared with non-diabetic controls. These findings do not support the hypothesis that exerciseinduced hypoglycaemia in patients with type 1 diabetes is due to suppression of changes in hepatic glycogen content. It is possible that diminished gluconeogenesis and/or increased peripheral glucose uptake might be important factors contributing to exercise-induced hypoglycaemia.

Acknowledgements We gratefully acknowledge The Special Trustees for Nottingham University Hospital for providing the funding to carry out the study. We would also like to thank S. Sarmad and S. Cordon for their assistance with the analytical work. We also acknowledge Eli Lily for providing a project grant. We are greatly indebted to all our volunteers for giving up their time to participate in this research study.

Duality of interest The authors declare that there is no duality of interest associated with this manuscript.

\section{References}

1. Dube MC, Valois P, Prud'homme D, Weisnagel SJ, Lavoie C (2006) Physical activity barriers in diabetes: development and validation of a new scale. Diabetes Res Clin Pract 72:20-27

2. Kemmer FW (1992) Prevention of hypoglycemia during exercise in type I diabetes. Diabetes Care 15:1732-1735

3. Tuominen JA, Karonen SL, Melamies L, Bolli G, Koivisto VA (1995) Exercise-induced hypoglycaemia in IDDM patients treated with a short-acting insulin analogue. Diabetologia 38:106-111

4. Tansey MJ, Tsalikian E, Beck RW et al (2006) The effects of aerobic exercise on glucose and counterregulatory hormone concentrations in children with type 1 diabetes. Diabetes Care 29:20-25

5. Wahren J, Felig P, Ahlborg G, Jorfeldt L (1971) Glucose metabolism during leg exercise in man. J Clin Invest 50:27152725

6. Jenkins AB, Chisholm DJ, James DE, Ho KY, Kraegen EW (1985) Exercise-induced hepatic glucose output is precisely sensitive to the rate of systemic glucose supply. Metabolism 34:431-436

7. Felig P, Wahren J (1975) Fuel homeostasis in exercise. N Engl J Med 293:1078-1084

8. Lavoie C, Ducros F, Bourque J, Langelier H, Chiasson JL (1997) Glucose metabolism during exercise in man: the role of insulin and glucagon in the regulation of hepatic glucose production and gluconeogenesis. Can J Physiol Pharmacol 75:26-35

9. Bjorkman O, Eriksson LS (1983) Splanchnic glucose metabolism during leg exercise in 60-hour-fasted human subjects. Am J Physiol 245:E443-E448

10. Rabasa-Lhoret R, Bourque J, Ducros F, Chiasson JL (2001) Guidelines for premeal insulin dose reduction for postprandial exercise of different intensities and durations in type 1 diabetic subjects treated intensively with a basal-bolus insulin regimen (ultralente-lispro). Diabetes Care 24:625-630

11. Zinman B, Murray FT, Vranic M et al (1977) Glucoregulation during moderate exercise in insulin treated diabetics. J Clin Endocrinol Metab 45:641-652 
12. DeFronzo RA, Ferrannini E, Hendler R, Felig P, Wahren J (1983) Regulation of splanchnic and peripheral glucose uptake by insulin and hyperglycemia in man. Diabetes 32:35-45

13. Wasserman DH, Spalding JA, Lacy DB, Colburn CA, Goldstein RE, Cherrington AD (1989) Glucagon is a primary controller of hepatic glycogenolysis and gluconeogenesis during muscular work. Am J Physiol 257:E108-E117

14. Hirsch IB, Marker JC, Smith LJ et al (1991) Insulin and glucagon in prevention of hypoglycemia during exercise in humans. Am J Physiol 260:E695-E704

15. Orskov L, Alberti KG, Mengel A et al (1991) Decreased hepatic glucagon responses in type 1 (insulin-dependent) diabetes mellitus. Diabetologia 34:521-526

16. Cline GW, Rothman DL, Magnusson I, Katz LD, Shulman GI (1994) ${ }^{13}$ C-nuclear magnetic resonance spectroscopy studies of hepatic glucose metabolism in normal subjects and subjects with insulin-dependent diabetes mellitus. J Clin Invest 94:2369-2376

17. Hwang JH, Perseghin G, Rothman DL et al (1995) Impaired net hepatic glycogen synthesis in insulin-dependent diabetic subjects during mixed meal ingestion. $\mathrm{A}{ }^{13} \mathrm{C}$ nuclear magnetic resonance spectroscopy study. J Clin Invest 95:783-787

18. Sindelar DK, Balcom JH, Chu CA, Neal DW, Cherrington AD (1996) A comparison of the effects of selective increases in peripheral or portal insulin on hepatic glucose production in the conscious dog. Diabetes 45:1594-1604

19. Sindelar DK, Chu CA, Neal DW, Cherrington AD (1997) Interaction of equal increments in arterial and portal vein insulin on hepatic glucose production in the dog. Am J Physiol 273: E972-E980

20. Sindelar DK, Chu CA, Rohlie M, Neal DW, Swift LL, Cherrington AD (1997) The role of fatty acids in mediating the effects of peripheral insulin on hepatic glucose production in the conscious dog. Diabetes 46:187-196

21. Sindelar DK, Chu CA, Venson P, Donahue EP, Neal DW, Cherrington AD (1998) Basal hepatic glucose production is regulated by the portal vein insulin concentration. Diabetes 47:523-529

22. Cherrington AD, Edgerton D, Sindelar DK (1998) The direct and indirect effects of insulin on hepatic glucose production in vivo. Diabetologia 41:987-996

23. Kishore P, Gabriely I, Cui MH et al (2006) Role of hepatic glycogen breakdown in defective counterregulation of hypoglycemia in intensively treated type 1 diabetes. Diabetes 55: 659-666

24. Petersen KF, Price TB, Bergeron R (2004) Regulation of net hepatic glycogenolysis and gluconeogenesis during exercise: impact of type 1 diabetes. J Clin Endocrinol Metab 89: 4656-4664

25. Chokkalingam K, Tsintzas K, Norton L, Jewell K, Macdonald IA, Mansell PI (2007) Exercise under hyperinsulinaemic conditions increases whole-body glucose disposal without affecting muscle glycogen utilisation in type 1 diabetes. Diabetologia 50:414-421

26. Borg GA (1982) Psychophysical bases of perceived exertion. Med Sci Sports Exerc 14:377-381

27. Frayn KN (1983) Calculation of substrate oxidation rates in vivo from gaseous exchange. J Appl Physiol 55:628-634
28. Williamson DH, Mellanby J, Krebs HA (1962) Enzymic determination of $\mathrm{D}(-)-\beta$-hydroxybutyric acid and acetoacetic acid in blood. Biochem J 82:90-96

29. Forster CD, Macdonald IA (1999) The assay of the catecholamine content of small volumes of human plasma. Biomed Chromatogr 13:209-215

30. Hoelzer DR, Dalsky GP, Clutter WE, Shah SD, Holloszy JO, Cryer PE (1986) Glucoregulation during exercise: hypoglycemia is prevented by redundant glucoregulatory systems, sympathochromaffin activation, and changes in islet hormone secretion. J Clin Invest 77:212-221

31. Cryer PE, Davis SN, Shamoon H (2003) Hypoglycemia in diabetes. Diabetes Care 26:1902-1912

32. Tuttle KR, Marker JC, Dalsky GP et al (1988) Glucagon, not insulin, may play a secondary role in defense against hypoglycemia during exercise. Am J Physiol 254:E713-E719

33. Wasserman DH, Williams PE, Lacy DB, Goldstein RE, Cherrington AD (1989) Exercise-induced fall in insulin and hepatic carbohydrate metabolism during muscular work. Am J Physiol 256:E500-E509

34. Wasserman DH, Lacy DB, Colburn CA, Bracy D, Cherrington AD (1991) Efficiency of compensation for absence of fall in insulin during exercise. Am J Physiol 261:E587-E597

35. Camacho RC, Pencek RR, Lacy DB, James FD, Wasserman DH (2004) Suppression of endogenous glucose production by mild hyperinsulinemia during exercise is determined predominantly by portal venous insulin. Diabetes 53:285-293

36. Raguso CA, Coggan AR, Gastaldelli A, Sidossis LS, Bastyr EJ 3rd, Wolfe RR (1995) Lipid and carbohydrate metabolism in IDDM during moderate and intense exercise. Diabetes 44:1066-1074

37. Simonson DC, Koivisto V, Sherwin RS et al (1984) Adrenergic blockade alters glucose kinetics during exercise in insulindependent diabetics. J Clin Invest 73:1648-1658

38. Purdon C, Brousson M, Nyveen SL et al (1993) The roles of insulin and catecholamines in the glucoregulatory response during intense exercise and early recovery in insulin-dependent diabetic and control subjects. J Clin Endocrinol Metab 76:566-573

39. Galassetti P, Tate D, Neill RA, Morrey S, Wasserman DH, Davis SN (2003) Effect of antecedent hypoglycemia on counterregulatory responses to subsequent euglycemic exercise in type 1 diabetes. Diabetes 52:1761-1769

40. Bischof MG, Bernroider E, Krssak M et al (2002) Hepatic glycogen metabolism in type 1 diabetes after long-term near normoglycemia. Diabetes 51:49-54

41. Petersen KF, Laurent D, Rothman DL, Cline GW, Shulman GI (1998) Mechanism by which glucose and insulin inhibit net hepatic glycogenolysis in humans. J Clin Invest 101:1203-1209

42. Wahren J, Hagenfeldt L, Felig P (1975) Splanchnic and leg exchange of glucose, amino acids, and free fatty acids during exercise in diabetes mellitus. J Clin Invest 55:1303-1314

43. Wahren J, Sato Y, Ostman J, Hagenfeldt L, Felig P (1984) Turnover and splanchnic metabolism of free fatty acids and ketones in insulin-dependent diabetics at rest and in response to exercise. J Clin Invest 73:1367-1376

44. Wasserman DH, Zinman B (1994) Exercise in individuals with IDDM. Diabetes Care 17:924-937 